\title{
Cádmio, cromo e chumbo em arroz comercializado no Rio Grande do Sul
}

\author{
Cadmium, chromium and lead in rice marketed in Rio Grande do Sul
}

\section{Christina Venzke Simões de Lima $^{\mathrm{I}^{*}}$ Lucélia Hoehne ${ }^{\mathrm{I}}$ Egon José Meurer $^{\mathrm{II}}$}

\section{- NOTA -}

\section{RESUMO}

O Rio Grande do Sul é o maior produtor nacional de arroz irrigado por alagamento do Brasil e algumas lavouras cultivam o arroz irrigado há mais de 100 anos. Nos cultivos, são utilizados insumos agrícolas, muitos dos quais apresentam metais pesados em sua composição. Essa preocupação leva em consideração principalmente os elementos não essenciais às plantas, como o $\mathrm{Pb}, \mathrm{Cr}$ e $\mathrm{Cd}$, e que oferecem risco à saúde humana. Esta pesquisa teve por objetivo quantificar os teores de $\mathrm{Cd}$, de $\mathrm{Cr}$ e de $\mathrm{Pb}$ no arroz vendido nos estabelecimentos comerciais do RS. As extrações dos elementos das amostras foram feitas pelo método USEPA 3050B. Para as quantificações dos metais, foi utilizada a Espectrometria de Emissão Óptica por Plasma Acoplado Indutivamente (ICP-OES). Os elementos quantificados nas amostras de grãos vendidos em estabelecimentos comerciais apresentaram-se dentro dos limites permitidos pela legislação.

Palavras-chave: metais pesados, contaminantes, alimento contaminado.

\section{ABSTRACT}

The state of Rio Grande do Sul (RS) is the largest producer of flood-irrigated rice in Brazil and some fields flooded rice are cultivated for more than 100 years. In rice production are being utilized various inputs, many of which have heavy metals in its composition. This preoccupation considers especially nonessential elements to plants such as $\mathrm{Pb}, \mathrm{Cr}$ and $\mathrm{Cd}$, which provide risks to human health. This study aimed to quantify the levels of $\mathrm{Cd}, \mathrm{Cr}$ and $\mathrm{Pb}$ in rice grains sold in markets retailers in RS. The elements were extracted from the samples through the USEPA 3050B method and quantified by Inductively Coupled Plasma Optical Emission Spectrometry (ICP-OES). The elements quantified in samples of grains rice sold in markets retailers were within the limits allowed by law.

Key words: heavy metals, contaminants, contaminated food.
O arroz é um alimento importante para a população mundial (FAO, 2013) e, em função disso, aspectos relacionados à sua produção e consumo devem ser continuamente monitorados e avaliados para que sua qualidade nutricional seja garantida. Segundo estudos de RUBIO et al. (1994), o arroz pode acumular naturalmente metais pesados em sua biomassa, não sendo de forma homogênea e, normalmente, apresentando maior acúmulo nas raízes, o que diminui o risco da entrada desses elementos na cadeia alimentar. No entanto, os teores de metais pesados nos tecidos das plantas dependem do $\mathrm{pH}$ do solo, da natureza do metal, do teor de matéria orgânica e da capacidade do solo em reter cátions (KABATA-PENDIAS \& PENDIAS, 2001).

A quantificação dos teores de metais pesados presentes em solos e disponíveis às plantas, principalmente em grãos, é fundamental na análise do risco de determinado ambiente. Há necessidade de se ampliar as pesquisas sobre a presença de metais pesados na produção vegetal, principalmente nos grãos, por plantas cultivadas em solos com potencial de contaminação. Diante desse fato, surgiu o interesse de analisar os grãos de arroz encontrados em estabelecimentos comerciais, com o intuito de confirmar ou não a segurança alimentar do consumo de arroz no Estado do Rio Grande do Sul. O objetivo deste estudo foi quantificar as concentrações de $\mathrm{Cd}, \mathrm{Cr}$ e $\mathrm{Pb}$ em amostras de arroz, de diferentes

\footnotetext{
'Programa de Pós-graduação em Biotecnologia, Centro Universitário UNIVATES (UNIVATES), Rua Avelino Tallini, 171, Bairro Universitário, 95900-000, Lajeado, RS, Brasil. E-mail: chris.solos@yahoo.com.br. *Autor para correspondência.

IIPrograma de Pós-graduação em Ciência do Solo, Universidade Federal do Rio Grande do Sul (UFRGS), Porto Alegre, RS, Brasil.
} 
empresas de beneficiamento do Rio Grande do Sul, comercializadas no Estado.

Existem aproximadamente 256 empresas de beneficiamento de arroz no Estado do Rio Grande do Sul, abrangendo todas as regiões orizícolas do Estado (AYRES et al., 2011). Destas, foram selecionadas doze amostras de arroz polido (Tabela 1), doze amostras de arroz parboilizado e três amostras de arroz integral (Tabela 2), englobando amostras de todas as regiões de cultivo orizícola do Estado do Rio Grande do Sul. As amostras foram adquiridas no comércio de cidades da Região Metropolitana de Porto Alegre - RS, de diferentes marcas e lotes.

Primeiramente, as amostras de grãos de arroz foram trituradas até passar inteiramente por uma peneira descartável de náilon de $0,15 \mathrm{~mm}$. Os teores totais de $\mathrm{Cd}, \mathrm{Cr}$ e $\mathrm{Pb}$ nas amostras foram determinados pelo método USEPA 3050B (USEPA, 1996). Este método foi selecionado por estar regulamentado na legislação brasileira, nas instruções normativas da Resolução 420 do CONAMA (2009).

A determinação dos teores de $\mathrm{Cd}, \mathrm{Cr}$ e $\mathrm{Pb}$ nos extratos das amostras de arroz foi feita por espectrometria de emissão atômica por plasma acoplado indutivamente (ICP-OES), modelo Optima 7300 DV, da Perkin Emer (comprimento de onda de 228, 802nm para Cd; 220,353nm para $\mathrm{Pb}$ e; 267 , $716 \mathrm{~nm}$ para $\mathrm{Cr}$ ), na Faculdade de Agronomia, da Universidade Federal do Rio Grande do Sul. Todas as análises foram feitas em triplicata. Para fins de controle do método, em cada bateria da análise, além do branco, fez-se uso de uma amostra padrão certificada (NIST SRM-695) para os elementos Cd, Cr e $\mathrm{Pb}$, que havia sido utilizada para certificação desses elementos em fertilizantes fosfatados, em estudo anterior. Os valores de certificação, os encontrados nas amostras e os percentuais de recuperação podem ser visualizados na tabela 3 .

As concentrações de $\mathrm{Cd}, \mathrm{Cr}$ e $\mathrm{Pb}$ encontradas nas amostras de arroz comercial (polido, parboilizado e integral) consumidas no Estado do RS estão apresentadas nas tabelas 1 e 2 . Os teores desses elementos, nas marcas de arroz analisadas, encontraram-se abaixo das descritas nos limites máximos de tolerância para os contaminantes inorgânicos $\mathrm{Cd}\left(0,4 \mathrm{mg} \mathrm{kg}^{-1}\right), \mathrm{Cr}(0,1 \mathrm{mg}$ $\left.\mathrm{kg}^{-1}\right)$ e $\mathrm{Pb}\left(0,2 \mathrm{mg} \mathrm{kg}^{-1}\right)$, em grãos de arroz, segundo a ANVISA (2015).

Poucos trabalhos de quantificação de metais pesados em arroz comercial no Brasil foram realizados. No Rio Grande do Sul, em estudo feito por POLETTI (2012), avaliando teores de metais pesados em arroz, verificou que as concentrações dos elementos $\mathrm{Cd}$ e $\mathrm{Pb}$ encontradas no estudo permaneceram abaixo dos limites de detecção do aparelho (ICP-OES) e, consequentemente, abaixo dos limites máximos permitidos (CODEX ALIMENTARIUS, 1995; ANVISA, 2014), corroborando este estudo.

Em análise de 25 amostras de arroz, disponíveis no mercado jamaicano, feita por Antoine et al. (2012), foram analisados 36 elementos essenciais, não essenciais e tóxicos, utilizando as técnicas de Espectroscopia de absorção atômica de chama (FAAS), Espectrometria de Emissão Óptica por Plasma Acoplado Indutivamente (ICPOES), análise por ativação neutrônica instrumental (INAA) e por fluorescência de raios $\mathrm{X}$ por reflexão

Tabela 1 - Código de identificação, municípios, regiões e a quantificação de $\mathrm{Cd}$, $\mathrm{Cr}$ e $\mathrm{Pb}$ nas amostras de arroz polido, comercializado no Estado do Rio Grande do Sul.

\begin{tabular}{|c|c|c|c|c|c|}
\hline \multirow{2}{*}{ Código de identificação } & \multirow{2}{*}{ Município do RS } & \multirow{2}{*}{ Região orizícola } & $\mathrm{Cd}^{*}$ & $\mathrm{Cr}^{*}$ & $\mathrm{~Pb}^{*}$ \\
\hline & & & & & \\
\hline $\mathrm{AC} 1$ & Pelotas & Sul & $<0,002$ & $<0,02$ & $<0,04$ \\
\hline $\mathrm{AC} 2$ & Palmares do Sul & Planície Costeira Externa & $<0,002$ & $<0,02$ & $<0,04$ \\
\hline $\mathrm{AC} 3$ & Camaquã & Planície Costeira Interna & $<0,002$ & $<0,02$ & $<0,04$ \\
\hline $\mathrm{AC} 4$ & São Gabriel & Campanha & $<0,002$ & $<0,02$ & $<0,04$ \\
\hline AC5 & Alegrete & Fronteira Oeste & $<0,002$ & $<0,02$ & $<0,04$ \\
\hline AC6 & Cachoeira do Sul & Depressão Central & $<0,002$ & $<0,02$ & $<0,04$ \\
\hline $\mathrm{AC7}$ & São Borja & Fronteira Oeste & $<0,002$ & $<0,02$ & $<0,04$ \\
\hline AC8 & Viamão & Planície Costeira Interna & $<0,002$ & $<0,02$ & $<0,04$ \\
\hline AC9 & S. Antônio da Patrulha & Planície Costeira Externa & $<0,002$ & $<0,02$ & $<0,04$ \\
\hline $\mathrm{AC} 10$ & Arroio Grande & Sul & $<0,002$ & $<0,02$ & $<0,04$ \\
\hline $\mathrm{AC} 11$ & Rosário do Sul & Campanha & $<0,002$ & $<0,02$ & $<0,04$ \\
\hline $\mathrm{AC} 12$ & Agudo & Depressão Central & $<0,002$ & $<0,02$ & $<0,04$ \\
\hline
\end{tabular}

AC - Arroz Polido.

*Limite de Detecção do aparelho (LD): $\mathrm{Cd}<0,002 \mathrm{mg} \mathrm{kg}^{-1}, \mathrm{~Pb}<0,02 \mathrm{mg} \mathrm{kg}^{-1}, \mathrm{Cr}<0,04 \mathrm{mg} \mathrm{kg}^{-1}$.

Ciência Rural, v.45, n.12, dez, 2015. 
Tabela 2 - Código de identificação, municípios, regiões e a quantificação de $\mathrm{Cd}, \mathrm{Cr}$ e $\mathrm{Pb}$ nas amostras de arroz parboilizado e integral, comercializados no Estado do Rio Grande do Sul.

\begin{tabular}{|c|c|c|c|c|c|}
\hline \multirow{2}{*}{ Código de identificação } & \multirow{2}{*}{ Município do RS } & \multirow{2}{*}{ Região orizícola } & $\mathrm{Cd}^{*}$ & $\mathrm{Cr}^{*}$ & $\mathrm{~Pb}^{*}$ \\
\hline & & & -------------- & 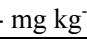 & \\
\hline $\mathrm{PC} 1$ & Camaquã & Planície Costeira Interna & $<0,002$ & $<0,02$ & $<0,04$ \\
\hline $\mathrm{PC} 2$ & Pelotas & Sul & $<0,002$ & $<0,02$ & $<0,04$ \\
\hline $\mathrm{PC} 3$ & S. Antônio da Patrulha & Planície Costeira Externa & $<0,002$ & $<0,02$ & $<0,04$ \\
\hline PC4 & Sentinela do Sul & Planície Costeira Interna & $<0,002$ & $<0,02$ & $<0,04$ \\
\hline PC5 & Itaqui & Fronteira Oeste & $<0,002$ & $<0,02$ & $<0,04$ \\
\hline PC6 & São Borja & Fronteira Oeste & $<0,002$ & $<0,02$ & $<0,04$ \\
\hline PC7 & Viamão & Planície Costeira Interna & $<0,002$ & $<0,02$ & $<0,04$ \\
\hline PC8 & Uruguaiana & Fronteira Oeste & $<0,002$ & $<0,02$ & $<0,04$ \\
\hline PC9 & Arroio Grande & Sul & $<0,002$ & $<0,02$ & $<0,04$ \\
\hline PC10 & Palmares do Sul & Planície Costeira Externa & $<0,002$ & $<0,02$ & $<0,04$ \\
\hline PC11 & Cachoeira do Sul & Depressão Central & $<0,002$ & $<0,02$ & $<0,04$ \\
\hline $\mathrm{PC} 12$ & Rosário do Sul & Campanha & $<0,002$ & $<0,02$ & $<0,04$ \\
\hline IC1 & Pelotas & Sul & $<0,002$ & $<0,02$ & $<0,04$ \\
\hline IC2 & Camaquã & Planície Costeira Interna & $<0,002$ & $<0,02$ & $<0,04$ \\
\hline IC3 & Eldorado do Sul & Planície Costeira Interna & $<0,002$ & $<0,02$ & $<0,04$ \\
\hline
\end{tabular}

PC - Arroz Parboilizado.

IC - Arroz Integral.

*Limite de Detecção do aparelho (LD): $\mathrm{Cd}<0,002 \mathrm{mg} \mathrm{kg}^{-1}, \mathrm{~Pb}<0,02 \mathrm{mg} \mathrm{kg}^{-1}, \mathrm{Cr}<0,04 \mathrm{mg} \mathrm{kg}^{-1}$.

total (TXRF). As concentrações médias de $\mathrm{Cd}$ no arroz branco e integral foram $<0,080 \mathrm{mg} \mathrm{kg}^{-1}$. Para $\mathrm{Cr}$, a média das concentrações no arroz branco e integral foram de $0,080 \mathrm{mg} \mathrm{kg}^{-1}$ e $0,157 \mathrm{mg} \mathrm{kg}^{-1}$, respectivamente. As concentrações dos metais pesados variam nos diferentes tecidos da planta e, em geral, os grãos contêm concentração menor do que as partes vegetativas da planta (BERTON, 2000). Isto pôde ser observado em estudo de SILVA et al. (2007), onde as plantas de arroz, em solos contaminados, restringiram a transferência de $\mathrm{Cd} \mathrm{e} \mathrm{Pb}$ do solo para os grãos. Outro estudo em que os teores de $\mathrm{Cd}, \mathrm{Cr}$ e $\mathrm{Pb}$ nos grãos de arroz se mantiveram abaixo dos limites permissíveis pelas normas reguladoras (CODEX ALIMENTARIUS, 1995; ANVISA, 2015) foi

Tabela 3 - Teores de $\mathrm{Cd}, \mathrm{Cr}$ e Pb certificados da amostra NISTSRM 695, teores encontrados por extração USEPA $3050 \mathrm{~B}$ e quantificados por ICP-OES e percentuais de recuperação dos elementos.

\begin{tabular}{llcc}
\hline \multirow{2}{*}{ Elementos } & $\begin{array}{c}\text { Teor NIST } \\
\text { SRM 695 }\end{array}$ & Teor quantificado & \\
& $-------------\mathrm{mg} \mathrm{kg}^{-1}$------------- & Recuperação \\
\hline $\mathrm{Cd}$ & $16,9 \pm 0,2$ & $15,6 \pm 01$ & 92,3 \\
$\mathrm{Cr}$ & $244 \pm 6$ & $238 \pm 3$ & 97,5 \\
$\mathrm{~Pb}$ & $273 \pm 17$ & $226 \pm 12$ & 82,8 \\
\hline
\end{tabular}

realizado por MORAES (2009), no qual foi simulada a entrada dos metais pesados no solo pela adição de diferentes doses e tipos de fertilizantes.

Estes cenários de ambientes contaminados diferem dos solos das plantações de arroz do Estado do Rio Grande do Sul, os quais foram avaliados por LIMA (2012) nas Planícies Costeiras Interna e Externa à Laguna dos Patos em concentrações baixas, não apresentando risco de contaminação. A partir dos dados levantados das lavouras das Planícies Costeiras do Rio Grande do Sul (LIMA, 2012), bem como dos grãos de arroz comercializados das regiões produtoras do Estado, afirma-se que não ocorre uma preocupação alarmante quanto aos teores de cádmio, cromo e chumbo nestes compartimentos. No entanto, é importante que ocorra, em estudos futuros, a avaliação dos demais solos das regiões orizícolas do Estado.

\section{AGRADECIMENTOS}

Ao Conselho Nacional de Desenvolvimento Científico e Tecnológico (CNPq), pelo financiamento da bolsa de doutorado para realização deste estudo.

\section{REFERÊNCIAS}

ANVISA (AGÊNCIA NACIONAL DE VIGILÂNCIA SANITÁRIA). Resolução - RDC n.42, de 29 de Agosto de 2013. Disponível em: <portal.anvisa.gov.br/wps/ 
wcm/connect/ 8100 bb 8040 eac 2 e 8 b5 590 b 79c ca $79 \mathrm{f} 4 \mathrm{cf} /$ $\mathrm{RDC}+\mathrm{n} \% \mathrm{C} 2 \% \mathrm{BA}+42 \_2013$ final.pdf?MOD $=\mathrm{AJPERES}>$. Acesso em: 10 mar. 2015.

AYRES, A.J. S. et al. Indústria Arrozeira no Rio Grande do Sul: notas sobre a localização da atividade e a estrutura produtiva. 2011. Disponível em: <http://www.pucrs.br/eventos/eeg/ trabalhos/54.doc $>$. Acesso em: mar. 2011.

BERTON, R. S. Riscos de contaminação do agroecossistema com metais pesados. In: BETTIOL, W.; CAMARGO, O.A. (Ed.). Impacto ambiental do uso agrícola do lodo de esgoto. Jaguariúna, SP: Embrapa Meio Ambiente, 2000. p.260.

CONAMA (CONSELHO NACIONAL DO MEIO AMBIENTE). Resolução CONAMA n. 420/2009. Disponível em: <http://www.mma. gov.br/port/conama/legiabre.cfm?codlegi=620>. Acesso em: 14 set. 2013.

CODEX ALIMENTARIUS. Norma geral para aditivos alimentares (GSFA) - CAC/STAN 192-1995 (última revisão) Disponível em: <http://www.codexalimentarius.net/gsfaonline/ index.html?lang=en>. Acesso em: 13 out. 2013.

FOOD AND AGRICULTURE ORGANIZATION OF THE UNITED NATIONS (FAO). Food Balance Sheet. 2001. Food and Agriculture Organization of the United Nations Statistical Databases (FAOSTAT), FAOSTAT Agriculture Data. Disponível em: <http://www.fao.org>. Acesso em: 10 out. 2013.

KABATA-PENDIAS, A.; PENDIAS, H. Trace elements in soils and plants. 3.ed. Boca Raton: CRC, 2001. 413p.
LIMA, C.V.S. de. Elementos-traço nos solos, águas e plantas de arroz irrigado do Rio Grande do Sul. 2012. 126f. Tese (Doutorado em Ciência do Solo) - Programa de Pós-graduação em Ciência do Solo, Faculdade de Agronomia, Universidade Federal do Rio Grande do Sul, Porto Alegre, RS.

MORAES, M.F. Micronutrientes e metais pesados tóxicos: do fertilizante ao produto agrícola. 2009. 108f. Tese (Doutorado em Ciências) - Programa de Pós-graduação em Ciências Área: Energia Nuclear na Agricultura e no Ambiente, Centro de Energia Nuclear na Agricultura, Universidade de São Paulo, Piracicaba, SP.

POLETTI, J. Quantificação de elementos-traço em arroz. 2012. 38f. Monografia (Trabalho de Conclusão de Curso) - Curso de Química Universidade Federal do Rio Grande do Sul, Instituto de Química, Porto Alegre, RS.

RUBIO, M.I. et al. Cadmium and Nickel accumulation in Rice plants, effects on mineral nutrition and possible interactions of abscisic acids. Plant Growth Regulation, Dordrecht, v.14, p.51-157, 1994.

SILVA, M.L. de S. et al. Concentração de metais pesados em grãos de plantas cultivadas em solo com diferentes níveis de contaminação. Pesquisa Agropecuária Brasileira, Brasília, v.42, n.4, p.527- 535, 2007.

USEPA. Method 3050 B. 1996. Disponível em: < http://www.epa. gov/wastes/hazard/testmethods/sw846/pdfs/3050b.pdf $>$. Acesso em: 20 mar. 2014 